VOL. 52 (1995) [247-252]

\title{
ON KY FAN'S COVERING THEOREMS FOR SIMPLEXES
}

\section{Chung-Wei Ha}

New covering properties of simplexes are obtained which extend and complement some covering theorems for simplexes of Fan.

\section{INTRODUCTION}

The covering theorems for simplexes of Sperner [7] and Knaster-KuratowskiMazurkiewicz [3] are well-known. These classical results were later generalized in Fan $[1,2]$. In this paper further covering properties of simplexes are proved which extend and complement those obtained by Fan. Our main result is Theorem 2 in Section 3. For its proof a key step is provided by Lemma 1 in Section 2 which is based on a basic congruence relation in integer labelling (see (4) below) given in Fan [2, Theorem 2] and an idea suggested in Sies $[\mathbf{5}, \mathbf{6}]$.

\section{A Lemma on INTEger Labelling}

We first recall some results of Fan on the integer labellings of pseudomanifolds which are needed to prove Lemma 1. For further references we refer to Fan [2].

For $x \in \mathbb{R}^{n+1}$, let $x_{1}, \ldots, x_{n+1}$ be the components of $x$, and let $I_{+}(x)=\{i$ : $\left.x_{i}>0\right\}, I_{0}(x)=\left\{i: x_{i}=0\right\}$. We denote an $n$-simplex by $\Sigma^{n}=\left\{x \in \mathbb{R}^{n+1}: x_{i} \geqslant\right.$ 0 for $\left.1 \leqslant i \leqslant n+1, \sum_{i=1}^{n+1} x_{i}=1\right\}$, and the boundary of $\Sigma^{n}$ by $\partial \Sigma^{n}$. Let $M^{n}$ be a triangulation of $\Sigma^{n}$. An admissible labelling $\phi$ of $M^{n}$ assigns to each vertex $v$ of $M^{n}$ an integer $\phi(v)$ satisfying

$$
\begin{aligned}
& \phi(v) \in\{ \pm 1, \ldots, \pm(n+1)\} \\
& \phi(v)+\phi(w) \neq 0 \quad \text { if } v, w \text { are adjacent vertices; } \\
& \phi(v)>0 \quad \text { if } v \text { is a vertex in } \partial \Sigma^{n}
\end{aligned}
$$

For any combination $\left(\varepsilon_{1}, \ldots, \varepsilon_{n+1}\right)$ of the signs $\varepsilon_{i}= \pm 1$, we denote by $\alpha\left(\varepsilon_{1} 1, \ldots\right.$, $\left.\varepsilon_{n+1}(n+1)\right)$ the number of those $n$-simplexes of $M^{n}$, each of which is labelled under $\phi$ by $\varepsilon_{1} 1, \ldots, \varepsilon_{n+1}(n+1)$ at its vertices, and denote by $\beta(1, \ldots, n)$ the number of those

Received 16th November, 1995

Copyright Clearance Centre, Inc. Serial-fee code: 0004-9729/95 \$A2.00+0.00. 
boundary $(n-1)$-simplexes of $M^{n}$, each of which is labelled under $\phi$ by $1, \ldots, n$ at its vertices. The congruence relation of Fan referred to in Section 1 asserts that for any combination $\left(\varepsilon_{1}, \ldots, \varepsilon_{n+1}\right)$ of the signs $\varepsilon_{i}= \pm 1$ with at least one $\varepsilon_{i}=-1$,

$$
\alpha\left(\varepsilon_{1} 1, \ldots, \varepsilon_{n+1}(n+1)\right) \equiv \alpha(1, \ldots, n+1)+\beta(1, \ldots, n) \quad \bmod 2 .
$$

We suppose further that $\phi$ satisfies one of the following boundary conditions:

$$
\begin{aligned}
& \phi(v) \in I_{+}(v) \quad \text { for all vertices } v \text { of } M^{n} \text { in } \partial \Sigma^{n} ; \\
& \phi(v) \in I_{0}(v) \quad \text { for all vertices } v \text { of } M^{n} \text { in } \partial \Sigma^{n} .
\end{aligned}
$$

If (5) holds, then it follows from the well-known Sperner's lemma [7] that

$$
\beta(1, \ldots, n) \equiv 1 \quad \bmod 2 .
$$

If we assume in addition to (6) that $M^{n}$ restricted to $\partial \Sigma^{n}$ is a further subdivision of the barycentric subdivision of $\partial \Sigma^{n}$, then as shown in Fan [2, Corollary 2], (7) also holds. In what follows, we shall make this additional assumption whenever (6) is assumed. Thus if $\phi$ satisfies either (5) or (6), then

$$
\alpha(-1, \ldots,-(n+1))+\alpha(1, \ldots, n+1) \equiv 1 \quad \bmod 2 .
$$

Lemma 1. Let $\phi, \psi$ be two admissible labellings of $M^{n}$ such that

$$
\begin{aligned}
& \psi(v) \neq \phi(w) \text { if } v, w \text { are vertices of } M^{n} \text { in } \partial \Sigma^{n} \text { such that either } \\
& v=w \text { or } v, w \text { are adjacent; } \\
& \psi(v)=-\phi(v) \text { if } v \text { is a vertex of } M^{n} \text { in } \Sigma^{n} \backslash \partial \Sigma^{n} .
\end{aligned}
$$

If either $\phi$ or $\psi$ satisfies one of the boundary conditions (5) and (6), then

$$
\alpha^{\phi}(-1, \ldots,-(n+1))+\alpha^{\psi}(-1, \ldots,-(n+1)) \equiv 1 \quad \bmod 2,
$$

where the superscript $\phi$ or $\psi$ indicates the labelling with respect to which the counting is performed.

Proof: Since the roles of $\phi$ and $\psi$ in both (9) and (10) are exchangable, it suffices to prove the theorem assuming that $\phi$ satisfies either (5) or (6). Then (8) with $\alpha$ specified to $\alpha^{\phi}$ holds true. We shall show more precisely that

$$
\alpha^{\phi}(1, \ldots, n+1)=\alpha^{\psi}(-1, \ldots,-(n+1)) .
$$

Clearly (12) holds if the equality sign is replaced by the sign $\geqslant$. It remains to show that if $\sigma$ is an $n$-simplex of $M^{n}$ labelled under $\phi$ by $1, \ldots, n+1$ at its vertices, then none of the vertices of $\sigma$ is in $\partial \Sigma^{n}$. Indeed, if $\sigma$ has a vertex $v$ in $\partial \Sigma^{n}$, then by (9) $\psi(v) \neq \phi(w)$ for any vertex $w$ of $\sigma$ in $\partial \Sigma^{n}$. Moreover, by (2) and (10) $\psi(v) \neq \phi(w)$ for any vertex $w$ of $\sigma$ in $\Sigma^{n} \backslash \partial \Sigma^{n}$. It follows that $\psi(v) \notin\{1, \ldots, n+1\}$ which is absurd. This proves (11). 


\section{Covering Theorems for Simplexes}

We denote by $I$ a subset of $\{1, \ldots, n+1\}$ and by $I^{\prime}$ the complement of $I$ in $\{1, \ldots, n+1\}$. For $A \subset \Sigma^{n}$, we denote $A^{\prime}=\Sigma^{n} \backslash A$. Our main result is the following

Theorem 2. Let $A_{i}, A_{-i}$ be $2(n+1)$ closed subsets of the $n$-simplex $\Sigma^{n}$. Consider the following statements:

$$
\begin{gathered}
\bigcup_{i=1}^{n+1}\left(A_{i} \cup A_{-i}\right)=\Sigma^{n}, \quad A_{i} \cap A_{-i}=\emptyset \quad \text { for } 1 \leqslant i \leqslant n+1 \\
\qquad \Sigma^{n} \subset \bigcup_{i=1}^{n+1} A_{-i} ; \\
x \in \bigcup_{i \in I_{+}(x)} A_{i} \quad \text { for all } x \in \partial \Sigma^{n} \\
x \in \bigcup_{i \in I_{0}(x)} A_{i} \text { for all } x \in \partial \Sigma^{n}
\end{gathered}
$$

If (13), (14) and either (15) or (16) are satisfied, then

$$
\left(\bigcap_{i \in I^{\prime}} A_{i}\right) \cap\left(\bigcap_{i \in I} A_{-i}\right) \neq \emptyset \text { if } \emptyset \neq I \neq\{1, \ldots, n+1\} \text {. }
$$

Moreover, at least one of the following

$$
\bigcap_{i=1}^{n+1} A_{i} \neq \emptyset
$$

holds.

Proof: Let $d$ be a metric of $\mathbb{R}^{n+1}, d_{i}=d\left(A_{i}, A_{-i}\right)$ for $1 \leqslant i \leqslant n+1$. We denote by $d_{0}$ the Lebesgue number of the family $\left\{A_{ \pm i}: 1 \leqslant i \leqslant n+1\right\}$ of $2(n+1)$ closed sets. Let $M^{n}$ be a triangulation of $\Sigma^{n}$ with mesh less than $\min \left\{d_{i}: 0 \leqslant i \leqslant n+1\right\}$. For a vertex $v$ of $M^{n}$, we define

$$
\phi(v)=i, \psi(v)=j \quad \text { if } v \in \partial \Sigma^{n}, v \in A_{i} \cap A_{-j}
$$

where $i, j \in\{1, \ldots, n+1\}$ and $i \in I_{+}(x)$ or $i \in I_{0}(x)$ according to whether (15) or (16) is assumed, and define

$$
\phi(v)=k, \psi(v)=-k \quad \text { if } v \in \Sigma^{n} \backslash \partial \Sigma^{n}, v \in A_{k},
$$


where $k \in\{ \pm 1, \ldots, \pm(n+1)\}$. It is straightforward to verify that $\phi, \psi$ are admissible labellings of $\Sigma^{n}$ satisfying (9) and (10).

It follows from Lemma 1 that either $\alpha^{\phi}(-1, \ldots,-(n+1)) \equiv 1 \bmod 2$ or $\alpha^{\psi}(-1, \ldots,-(n+1)) \equiv 1 \bmod 2$. For $I \neq \emptyset$, let $\left(\varepsilon_{1}, \ldots \varepsilon_{n+1}\right)$ be so chosen that $\varepsilon_{i}=-1$ if $i \in I$ and $\varepsilon_{i}=1$ if $i \in I^{\prime}$. If the first case is true, then by (4) $\alpha^{\phi}\left(\varepsilon_{1} 1, \ldots, \varepsilon_{n+1}(n+1)\right) \equiv 1 \bmod 2$. Thus there exists an $n$-simplex $\sigma=v_{1}, \ldots, v_{n+1}$ of $M^{n}$ with $\phi\left(v_{i}\right)=\varepsilon_{i} i$, that is, $v_{i} \in A_{\varepsilon_{i} i}$, for $1 \leqslant i \leqslant n+1$. Since the mesh of $M^{n}$ is less than the Lebesgue number $d_{0}$, it follows that (17), (19) hold. If the second case is true, then by (4) again $\alpha^{\psi}\left(\varepsilon_{1} 1, \ldots, \varepsilon_{n+1}(n+1)\right) \equiv 1 \bmod 2$. It follows from a similar argument that (17), (18) hold. This completes the proof of the theorem.

If we assume $\bigcap_{i=1}^{n+1} A_{i}=\emptyset$ in Theorem 2, then the condition (14) is superfluous, since in this case obviously $\alpha^{\phi}(1, \ldots, n+1)=0$ and so by $(8) \alpha^{\phi}(-1, \ldots,-(n+1)) \equiv 1$ mod 2, where $\phi$ is the admissible labelling defined in the proof of Theorem 2. Thus we obtain (17), (19) without assuming (14). This is precisely the assertion of Fan [2, Theorems 3 or 4] according to whether (15) or (16) is assumed.

As an immediate consequence of Theorem 2, we obtain in Theorem 4 below another covering property of $\Sigma^{n}$. We first give a simple lemma which is also useful in another situation as noted at the end of Section 3 (see also Shih [4, Theorem 1]).

Lemma 3. Let $X$ be a compact metric space, $A_{i}(1 \leqslant i \leqslant m)$ be open subsets of $X$. Let $\Gamma$ be a family of nonempty subsets of $\{1, \ldots, m\}$ and for $\gamma \in \Gamma$ let $X_{\gamma}$ be a closed subset of $X$ such that for all $\gamma \in \Gamma$

$$
X_{\gamma} \subset \bigcup\left\{A_{i}: i \in \gamma\right\}
$$

Then there exist closed subsets $B_{i}(1 \leqslant i \leqslant m)$ such that $B_{i} \subset A_{i}$ for $1 \leqslant i \leqslant m$ and for all $\gamma \in \Gamma$

$$
X_{\gamma} \subset \bigcup\left\{B_{i}: i \in \gamma\right\}
$$

Proof: Let $d$ be the metric of $X$. We define $f_{i}(x)=d\left(x, A_{i}^{\prime}\right)$ for $1 \leqslant i \leqslant m$, $f(x)=\max _{1 \leqslant i \leqslant m} f_{i}(x)$. For each $\gamma \in \Gamma, f(x)>0$ on the compact set $X_{\gamma}$ and so there exists $\varepsilon_{\gamma}>0$ such that $f(x) \geqslant \varepsilon_{\gamma}$ for $x \in X_{\gamma}$. Let $\varepsilon=\min \left\{\varepsilon_{\gamma}: \gamma \in \Gamma\right\}$. Then the sets $B_{i}=\left\{x \in A_{i}: f_{i}(x) \geqslant \varepsilon\right\}(1 \leqslant i \leqslant m)$ have the property required.

TheOREM 4. Let $A_{i}(1 \leqslant i \leqslant n+1)$ be $n+1$ closed subsets of $\Sigma^{n}$ and let 
$C, D \subset \Sigma^{n}$ such that $D$ is closed and $C \subset D$. Consider the following statements:

$$
\begin{gathered}
C \cup \bigcup_{i=1}^{n+1} A_{i}=\Sigma^{n}, \quad D \cap \bigcap_{i=1}^{n+1} A_{i}=\emptyset \\
\partial \Sigma^{n} \subset \bigcup_{i=1}^{n+1} A_{i}^{\prime} .
\end{gathered}
$$

If (20), (21) and either (15) or (16) are satisfied, then

$$
\left(\bigcap_{i \in I^{\prime}} A_{i}\right) \cap\left(\bigcap_{i \in I} A_{i}^{\prime}\right) \neq \emptyset \quad \text { if } \emptyset \neq I \neq\{1, \ldots, n+1\}
$$

Moreover, at least one of (18) and

$$
\bigcap_{i=1}^{n+1} A_{i}^{\prime} \neq \emptyset
$$

holds.

Proof: It follows from the the second equality in (20) that $\Sigma^{n}=D^{\prime} \cup \bigcup_{i=1}^{n+1} A_{i}^{\prime}$. By Lemma 3 there exist closed subsets $A_{-i}(1 \leqslant i \leqslant n+1)$ of $\Sigma^{n}$ such that $A_{-i} \subset A_{i}^{\prime}$ for $1 \leqslant i \leqslant n+1$ and

$$
\Sigma^{n}=D^{\prime} \cup \bigcup_{i=1}^{n+1} A_{-i}, \quad \partial \Sigma^{n} \subset \bigcup_{i=1}^{n+1} A_{-i}
$$

It follows from the first equality in (20) that $\bigcup_{i=1}^{n+1} A_{i} \supset C^{\prime} \supset D^{\prime}$ and so

$$
\Sigma^{n}=\bigcup_{i=1}^{n+1}\left(A_{i} \cup A_{-i}\right)
$$

The result follows by applying Theorem 2 to the $2(n+1)$ closed sets $A_{i}, A_{-i}$ $(1 \leqslant i \leqslant n+1)$.

Some interesting special cases of Theorem 4 are obtained by properly specifying the sets $C$ and $D$. Note that (21) is trivially satisfied if $\bigcap_{i=1}^{n+1} A_{i}=\emptyset$. Thus if we assume $\bigcup_{i=1}^{n+1} A_{i}=\Sigma^{n}$ in addition to either (15) or (16), then it follows that $\bigcap_{i=1}^{n+1} A_{i} \neq \emptyset$. This is the covering property of Knaster-Kuratowski-Mazurkiewicz or Sperner according to 
whether (15) or (16) is assumed. On the other hand, let us assume further in Theorem 4 that $C$ is closed and $\bigcap_{i=1}^{n+1} A_{i}=\emptyset$, and write

$$
\Sigma^{n}=\bigcup_{i=1}^{n+1}\left(A_{i} \cup\left(C \cap A_{-i}\right)\right)
$$

in place of (22). Since in this case the condition (14) is superfluous for Theorem 2 to hold as remarked following the proof of Theorem 2 , we obtain

$$
C \cap\left(\bigcap_{i \in I^{\prime}} A_{i}\right) \cap\left(\bigcap_{i \in I} A_{i}^{\prime}\right) \neq \emptyset \quad \text { if } I \neq \emptyset
$$

a conclusion stronger than Theorem 4 would imply. This is precisely the assertion of Fan [2, Theorem 5] or Fan [1, Theorem 1] according to whether (15) or (16) is assumed. We note that the condition (20) is equivalent to the condition

$$
\bigcap_{i=1}^{n+1} A_{i} \subset \operatorname{int} \bigcup_{i=1}^{n+1} A_{i}
$$

not involving the sets $C, D$, where int denotes the interior relative to $\Sigma^{n}$.

Finally we see by using Lemma 3 that Theorems 2 and 4 remain valid when the sets $A_{i}, A_{-i}(1 \leqslant i \leqslant n+1)$ in Theorem 2 and the sets $A_{i}(1 \leqslant i \leqslant n+1), C$ in Theorem 4 are assumed to be open. Moreover, (23) also holds if we assume further that $\bigcap_{i=1}^{n+1} A_{i}=\emptyset$.

\section{REFERENCES}

[1] K. Fan, 'A covering property of simplexes', Math. Scand. 22 (1968), 17-20.

[2] K. Fan, 'A combinatorial property of pseudomanifolds and covering properties of simplexes', J. Math. Anal. Appl. 31 (1970), 68-80.

[3] B. Knaster, C. Kuratowski and S. Mazurkiewicz, 'Ein Beweis des Fixpunktsatzes für n-dimensionale Simplexe', Fund. Math. 14 (1929), 132-173.

[4] M.H. Shih, 'Covering properties of convex sets', Bull. London Math. Soc. 18 (1986), 57-59.

[5] H. Sies, 'Topological degree and Sperner's lemma', Fund. Math. 118 (1983), 135-149.

[6] H. Sies, 'A covering theorem for simplexes', J. Math. Anal. Appl. 98 (1984), 599-602.

[7] E. Sperner, 'Neuer Beweis für die Invarianz der Dimensionszahl und des Gebietes', $A b h$. Math. Sem. Univ. Hamburg 6 (1928), 265-272.

Department of Mathematics National Tsing Hua University Hsin Chu Taiwan 